\title{
Hybrid Technique for Enhancing Underwater Image in blurry conditions
}

\author{
Chrispin Jiji ${ }^{*}, 1$, Nagaraj Ramrao ${ }^{2}$ \\ ${ }^{1}$ Kalasalingam Academy of Research and Education, Krishnankoil-626126, Tamilnadu, India \\ ${ }^{2}$ Department of ECE, The Oxford College of Engineering, Bengaluru-560068, Karnataka, India
}

\begin{tabular}{l} 
A R T I C L E I N F O \\
\hline Article history: \\
Received:03 March, 2019 \\
Accepted:10 April, 2019 \\
Online: 17 April, 2019 \\
\hline Keywords: \\
Image Restoration \\
Image Enhancement \\
Color Adjustment \\
Hybrid Technique \\
\end{tabular}

\begin{abstract}
A B S T R A C T
Enhancing underwater visualization using hybrid technique is generally employed into oceanic production. Through growing oceanic learning, undersea processing has drawn extra importance owing towards necessary task of picture towards attaining data. Although, suitable to reality of dust-like constituent and beam reduction, undersea descriptions continually experience small contrast and color alteration. In this paper, we estimate submerged beam transmission progression also intend an effectual means to defeat the backscatter trouble. Our scheme generally includes three steps; first, we reconstruct the picture using adaptive regularization. Second, we separate the reconstructed picture with weighted decomposition; third, we exploit color adjustment along with dehazed process via gradient guided filter towards holding dual mechanisms independently; at last, re-establish fine effect, we use hybrid technique for enhancing the picture. The tentative outcome illustrates that our proposed process extensively get better quality of unclear submerged descriptions. In common, our proposed method verified as well-performed and effectual than existing technique.
\end{abstract}

\section{Introduction}

In the modern years, scientists and researchers have exposed their main concern in capturing the aquatic life beneath the water. Although the statement that $70 \%$ of the globe encloses water includes enormous energy, abundant mineral assets and biological resources. The living beneath the water is still not as much look at. Conversely, challenges related by capturing descriptions beneath the water have been complex to defeat, owing to carry-over haze and color cast. The muddy nature of water suitable to process element such as mineral deposits, sand and plankton, showed a huge difficulty in undersea look at region. These elements set up lack of clarity in undersea descriptions which spoils the clearness and ocular analysis of the picture and in turn, inappropriate for any advance use. Researchers have finished that the spreading and absorption property of beam underneath are the chief basis for this deformation [1]. While water is roughly 800 times denser than air, so when beam penetrate from air to water, it come across reflection fact at the surface owing to which only unfair measure of beam go through water. Afterwards the beam undergoes spreading cause when it strikes and element and minerals suspend in water. Scattering diverges the beam in dissimilar directions which diminish the measure of beam falling on the object captured

${ }^{*}$ Chrispin Jiji, 8951627124 \& chrispinjij@gmail.com underneath. The end effect of spreading outcome is that the undersea captured descriptions are dim in look [2]. A further problem is causal to lack of clarity of picture that the water molecules take up certain measure of beam when beam hit on them. Because the mass of water is larger than air, it employs dissimilar absorption possessions for diverse beam wavelengths, so the color of undersea descriptions is typically deformed. Generally, red part vanishes first as of its highest wavelength, where blue beam, by small wavelength, convey the greatest path beneath water. Therefore, undersea descriptions are often dominated via blue color. In common, color cast and beam scattering reason deviate color along with contrast degradation into descriptions attained undersea as shown in Figure 1.

In contrast, our system uses dual phases specifically Adaptive Regularization for restoration and Hybrid technique intended for improving its quality. In this work we suggested for merging the thought mainly dictionary knowledge by structural cluster along with picture restoring functions.

Our advance establishes novel regularization to beat a superior stability among local and non-local strength. Generally, our algorithm interprets learning through local and non-local similarity give inference of each picture elements. The restored picture decomposes into dissimilar elements for signifying their 


\section{Jiji et al. / Advances in Science, Technology and Engineering Systems Journal Vol. 4, No. 2, 340-350 (2019)}

basic depiction, later uses diverse development for every part individually along with hybrid technique introduced for rebuilding enhanced picture. The projected process is close towards or even slightly superior to the state-of-the-arts. Tentative results exposed that projected process extensively outperforms many important process in existing arts.

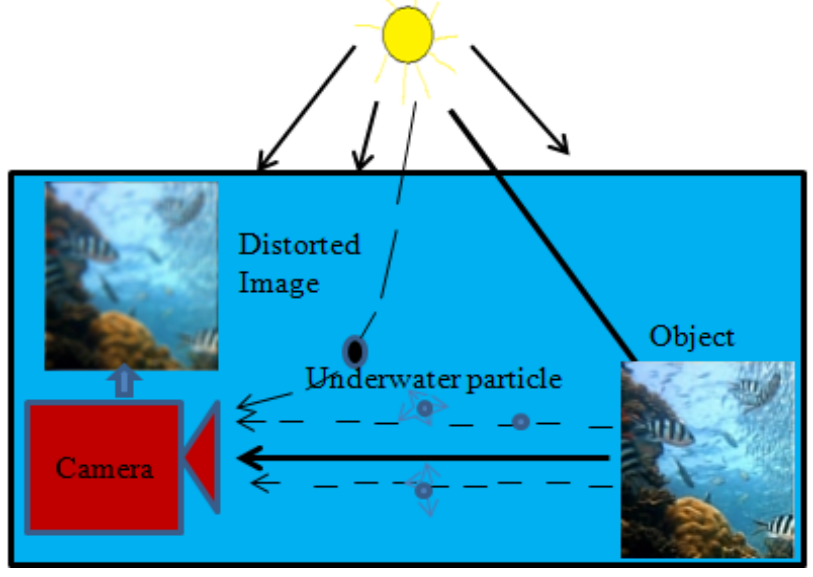

Figure 1: Underwater Imaging System

The summary of this work described as follow. Section 2 portray existing art. Section 3 projects hybrid technique using gradient guided filter for enhancing undersea descriptions. Section 4 illustrates numerical experiments. At last, Section 5 concludes this work.

\section{Existing art}

Enhancement system for undersea degradation typically explore the undersea optical form and balance bad possessions caused by water along with elements, or simply via undersea processing process for enhancing unclear imagery. State-of-arts are generally considered meant for single image enhancement, since multi-images may limit performance choice, slow down the processing speed. Instinctively, handling this concern well, undersea optical form must consider and investigated at first. Among different undersea imaging methods, the representation method [3], give a complete undersea optical form, within which beam transmission path, beam reduction relation of dissimilar color channel, vertical as well as horizontal deepness of undersea surroundings, non-natural beam possessions and etc are in use. This process is entirely with real form and meet outstanding for a few undersea problems, conversely, its evaluation procedure is difficult and time overwhelming, which is hard to meet, but it gives a full-scale study of undersea surroundings. Fusion-based method, which combines processing outcome by color correction system and histogram equalization method, it also makes a superior outcome for several submerged environments, though, it merely uses scheme of image processing as well as ignoring submarine features, hence ending results undergo more or less fog, data defeat as well as over-enhancement difficulty. To explain these problems well, both processes combined and projected a new method. Semi-inverse approach in [4] employed towards perceiving foggy region and then expected atmospheric rate in plain areas. At last, they meet enhanced picture using a simple weighted fusion.

For few picture, particularly scenes underneath, mist exposure using semi-inverse system will be unsuccessful suitable to the $\underline{\text { www.astesj.com }}$ small dissimilarity among true and the inverse picture. This means if hazy region exposure not succeeds and obtains a bad performance. To solve this problem, a simple fusion-based dehazed method using a dissimilar descriptions also resultant from true picture [5], [6]. The primary picture estimate white balance process, and the next input picture through a simple linear change for enhancing contrast of hazy picture. At last, the pixelfusion scheme uses three weight maps for combining that imagery for enhancing visibility of foggy picture also extended fusionbased scheme [7]. Fusion-based dehazed procedure is easy, speedy as well as meets alike result as the DCP scheme in [8] and [9]. But it is not succeeding when the picture includes inhomogeneous haze. The cause is that this process did not take into account the deepness data of a hazy picture. The dual descriptions attained by gamma correction by various scales for sandstorm enhancement [10]. The enhanced fusion-based approach using various descriptions uses single picture dehazed scheme in [11]. Fusion approach is new and efficient intended for single picture defogging. The primary picture is for enhancing dissimilarity; in addition, later picture mainly for compensating color deformation as well as decrease halo effect plus noise. While above techniques are fast as well as simple, they cannot make excellent picture. Amusingly, the multi-scale progression can be approximated with well-organized and visually satisfying single-scale process [12]. HR fusion in [13] has to be measured as a possible balance, useful to our process when high resolution is required.

The algorithm [14] are effective methods with low computational complexity also great performance, which stretch histograms of picture for improving the dynamic range compress the display area towards enhancing dissimilarity, but these methods often cause over-enhancement, amplify the noise and show poor effect on color correction. Owing towards the drawbacks of single image processing, some relatively complicated methods are proposed. Meanwhile, [15] proposes a retinex-based approach to deal with undersea issues, however, performance of this system is not distinct, color cast as well as contrast degradation still exist. The system may guess a uniform $[16,17]$ or an extra reasonable nonuniform [18] background-radiance. Enhancement technique eliminates the colour cast $[19,20]$ with overall white balancing. This work is extensive to the effort primarily obtained through ICCIC [21]. Moreover, the projected work is for Enhancing Underwater Image in blurry conditions using hybrid technique. Revised outcome much improves lighting plus color cast trouble during submerged descriptions. Excellence process not essential to resolve substantial type of corrupted representation, other than fairly enhances dissimilarity also gets superior picture excellence as individual illustration view. Experimental results show good performance meant for underneath representation.

\subsection{Motivation}

Underwater distorted descriptions typically to explore optical model also compensating bad effects caused by underwater element uses processing scheme to restore performance of distorted images. The state-of-arts are mostly designed for singleimage enhancement, since multi-images may limit performance range and slow down processing speed. Towards handling this problem well, initially we considered Image re-establishment process. The physical system of ocular imaging, reverse 


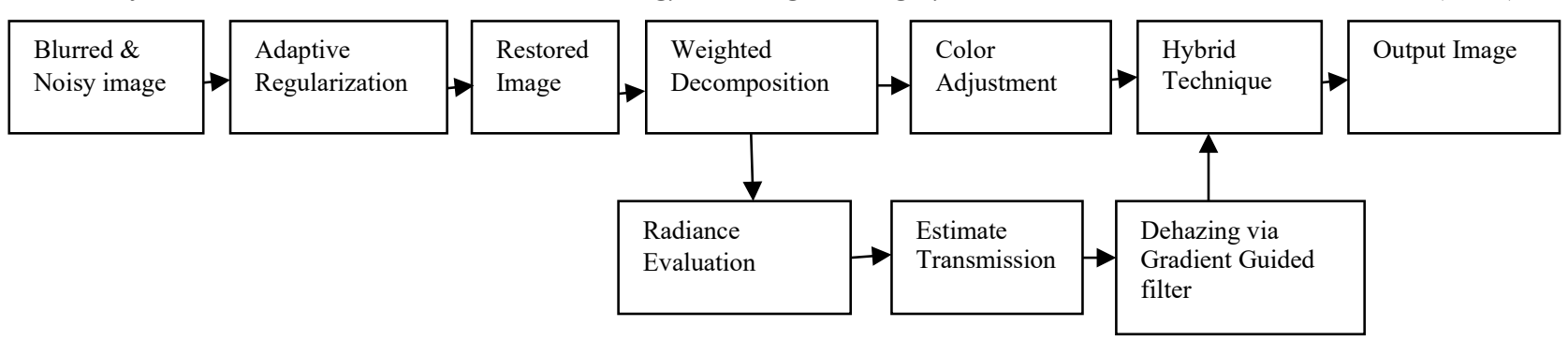

Figure 2: Block diagram of Proposed Method

degradation process and balance distortion to get clear imagery but it has small visibility and color cast problem. Image enhancement process do not get the source of picture degradation into account, but mainly use targeted version to get better dissimilarity, detail, and ocular possessions. It provides complete undersea ocular form, where the illuminated transmission path, beam reduction of dissimilar color path taken into concern totally based on physical model and achieves excellent for some underwater backscatter issues. However, its evaluation development is difficult and time-consuming, but it gives fullscale analysis of undersea situation. To enhance dissimilarity, visibility, color spread of restored imagery, fusion-based model which boost picture by fusing several input imageries have received much attention. The processing results by color constancy and dehazed algorithm, generates good results. However, it only uses technique of image processing and so its results suffer from more or less haze, information failure as well as over-enhancement problem.

In order to solve this issue well, we proposed a Hybrid Technique for Enhancing Underwater Image in blurry conditions by joining dehazed technique via gradient guided filter with color balance of the restored imagery.

\section{Proposed Method}

As depicted in Figure 2 our new scheme primarily to Enhance Undersea picture in blurry conditions using hybrid technique. Initially, we recreate the picture using adaptive regularization. Secondly, we split reconstructed picture using weighted decomposition. Thirdly, improve contrast and texture information using Dehazing via gradient guided filter. Here we estimate global radiance by obtaining average rate vector as brightest rate, transmission map evaluation mainly for enhancing texture feature. Fourth, a color adjustment towards enhancing color. At last, we used hybrid technique for obtaining better picture by computing the mechanisms of dual weights in addition to efficient.

\subsection{Adaptive Regularization for Restoration}

For an image $d \in P^{H X W}$, we believe patch based depiction $d_{m}=P d \in P^{N}, m=1,2, \ldots . . N$ where $P$ indicates a parameter to extract patch $d_{m}$ as of $d$. Suppose a known dictionary $\varphi_{m}$ meant for $d_{m}$, we get $d_{m}=\varphi_{m} \alpha_{m}$ entails $\alpha_{m}$ as sparse depiction of $d$. In particular, restoration describes

$$
D=\left(\sum_{i=1}^{N} P_{i}^{T} P_{i}\right)^{-1}\left(\sum_{i} P_{i}^{T} d_{i}\right)
$$

The iterative shrinkage key towards 11-estimation is widely used in previous art. Since the evaluation of $\hat{d}$ get simplified by convergence, trained by a novel dictionary. At present let us include adaptive regularization term [22,23]. For every patch $d_{m}$ k-nearest-neighbors patch signify $d_{m}^{(1)}, \ldots . . d_{m}^{(k)}$. The weighted guess is representing

$$
d_{i}^{\wedge}=\sum_{t=1}^{k} w_{m, t} D_{i}^{(t)}, w_{m, t}=\exp \left(-\left\|D_{m}-D_{m}^{(t)}\right\| / h\right)
$$

where $h$ stands for constant to manage the window. Since (2) local form by picture roughly considered with

$$
D_{L}(d)=\sum_{m=1}^{M}\left\|d_{m}-a_{k_{m}}^{T} \chi_{m}\right\|_{2}^{2}
$$

In matrix type (3) represents

$$
D_{L}(d)=\sum_{m=1}^{M}\left\|(I-V) d_{m}\right\|_{2}^{2}=\|(I-V) \varphi \alpha\|_{2}^{2}
$$

where $I$ specify identity matrix and

$$
V(m, n)=\left\{\begin{array}{l}
a_{i}, \text { if } d_{n} \text { is an element of } \chi_{m}, a_{m} \in a_{k_{m}} \\
0, \text { otherwise }
\end{array}\right.
$$

It follows from (2) that the nonlocal similarity in a picture roughly considered through

$$
D_{N L}(d)=\sum_{m=1}^{M}\left\|d_{m}-\sum_{t=1}^{k} w_{m, t} d_{m}^{(t)}\right\|_{2}^{2}
$$

which can be written more efficiently in a matrix type as

$$
D_{N L}(d)=\sum_{m=1}^{M}\left\|(I-W) d_{m}\right\|_{2}^{2}=\|(I-W) \varphi \alpha\|_{2}^{2}
$$

Where $I$ stands for the identity and

$$
W(m, l)=\left\{\begin{array}{l}
b_{i}^{l}, \text { if } d_{m}^{l} \text { is an element of } \beta_{m}, b_{m}^{l} \in b_{m} \\
0, \text { otherwise }
\end{array}\right.
$$

Joining (4) and (7), we have the next novel regularization as

$$
\hat{\alpha}=\arg \min \left\{\|d-H \varphi \alpha\|_{2}^{2}+\sum_{m=1}^{M} \sum_{n=1}^{N} \lambda_{m n}\left|\alpha_{m, n}\right|\right\}+\gamma\|(I-V) \varphi \alpha\|_{2}^{2}+\eta\|(I-W) \varphi \alpha\|_{2}^{2}(9)
$$




\section{Jiji et al. / Advances in Science, Technology and Engineering Systems Journal Vol. 4, No. 2, 340-350 (2019)}

where a novel regularization factor $\eta$ manage stability among local variation with nonlocal strength. The nonlocal regularization term not only imposes nonlocal similarity however as well not directly assist the evaluation of signal variance essential with $\lambda_{m n}$ as $\lambda_{m n}=\frac{2 \sqrt{2} \sigma_{w}^{2}}{\left|\sigma_{m, n}^{\wedge}\right|+\varepsilon}$ where $\sigma_{m, n}$ and $\sigma_{w}$ signifies the variance of signal and noise correspondingly.

\subsection{Weighted Decomposition}

Weighted decomposition used to separate imagery into various parts and then perform several schemes according to their characteristics. Techniques like wavelet transform and weighted decomposition are most well-liked process. Former scheme is time consuming and cannot take out the characteristics. Later on initiate a weighted decomposition means, which divide true picture into dual part, one conveys the illuminant part as another states the reflectance part of picture as:

$$
I_{c}(d)=I_{c}^{R}(d)+I_{c}^{I}(d)
$$

where $c \in\{r, g, b\}$ signifies every color part of picture, $I_{c}^{R}(d)$ denotes reflectance part and $I_{c}^{I}(d)$ represents illumination part.

$$
\begin{aligned}
& I_{c}^{R}(d)=\Upsilon \cdot I_{c}(d) \\
& I_{c}^{I}(d)=(1-\Upsilon) \cdot I_{c}(d)
\end{aligned}
$$

where $\Upsilon$ denotes weighted factor which improve the dissimilarity of reflectance part for removing backscatter as

$$
\Upsilon=\xi \cdot \frac{I_{c}(d)}{I_{c}^{\max }}
$$

Where $I_{c}^{\max }$ denotes maximal pel rate of color, $\xi$ signify a direct cause by resolving reflectance weight, $\xi \in(0,1)$, if $\xi=0$, the entire picture treated as illuminate part, while $\xi=1$, the entire picture is denoted by reflectance part.

The backscatter outcome simply exists with illuminate part, whereas reflectance part simply experiences as of color deformation.

\subsection{Radiance Evaluation}

To approximate background beam, a supreme approach is to select a picture element, while color deformation and dissimilarity degradation are distance dependent. Though, in this system, scenes are vivid than surrounding beams, may perhaps an unwanted collection outcome. To get precise outcome, we exploit a hierarchical searching means using quad-tree subdivision [24]. Initially, the picture broken up into four identical rectangular areas, after that used for all area by estimating mean rate subtracted from standard deviation as

$$
\text { Score }_{l}=\frac{1}{3 N} \sum_{c \in\{r, g, b\}} \sum_{d=1}^{N} I_{l}^{c}(d)-\frac{1}{3} \sum_{c \in\{r, g, b\}} \sqrt[2]{\frac{\sum_{d=1}^{N}\left(I_{l}^{c}(d)-\bar{I}_{l}\right)^{2}}{N}}
$$

Where $l=1,2,3,4$ stand for four image areas, $N$ denotes the picture element number contained by the area, $I_{l}^{c}(d)$ signifies picture element of $d$ rate $c$ section in $l$ area, $I_{l}$ represents mean picture element rate of $c$ part in $l$ area. Later, we choose the area by least variance, as well as split into quadrant areas. This procedure continues until the size is smaller than threshold. The average rate attained as surrounding beam and considered as brightest rate with the complete representation.

\subsection{Estimate Transmission}

The transmission rate cannot be selected randomly; dissimilarity improvement with data defeat lessening must chose keen on concern. First, dissimilarity improvement cost task, $E_{c}$, with data loss cost task $E_{i}$ reduce dual function concurrently, while dissimilarity improvement cost as negative summation of $C_{M S E}$ of every color channels, furthermore data defeat cost as summation of square of reduced picture elements as

$$
\begin{gathered}
E_{c}=-\sum_{c \in\{r, g, b\}} \sum_{d=1}^{N} \frac{\left(J_{c}^{T}(d)-\bar{J}_{c}\right)^{2}}{N}=-\sum_{c \in\{r, g, b\}} \sum_{d=1}^{N} \frac{\left(I_{c}^{T}(d)-\bar{I}_{c}^{T}\right)^{2}}{N . t(d)^{2}} \\
E_{i}=-\sum_{c \in\{r, g, b\}} \sum_{d=1}^{N}\left\{\left(\min \left\{0, J_{c}^{T}(d)\right\}\right)^{2}+\left(\max \left\{0, J_{c}^{T}(d)-255\right\}\right)^{2}\right\}
\end{gathered}
$$

At last, for every local region the best transmission rate $t(d)$ is approximated by reducing the later task as,

$$
E=E_{c}+\gamma \cdot E_{i}
$$

where $\gamma$ denotes weighted factor to organize the control of data loss cost.

\subsection{Dehazing via Gradient Guided Filter}

The Guided filter in [25] refine transmission map but cannot signify fine next to a few boundaries. While there might be a few halos in the descriptions. The halos decrease the visual excellence of the resultant descriptions. Hence Gradient guided filtering is an effectual and process transmission map, use input picture as guidance can have executed by linear system as

$$
(L+\lambda \cdot U) \cdot t=\lambda \cdot \tilde{t}
$$

where $L$ represents matting Laplacian matrix, $U$ denotes identity matrix, and $\lambda$ signify regularization factor. The Laplacian matrix represents 


\section{Jiji et al. / Advances in Science, Technology and Engineering Systems Journal Vol. 4, No. 2, 340-350 (2019)}

$$
L_{i j}=|\omega|\left(\delta_{i j}-W_{i j}\right)
$$

where $|\omega|$ stands for number of picture elements in a block, $\delta_{i j}$ denotes Kronecker delta, and $W_{i j}$ represents gradient guided filter [26] kernel weight as:

$$
W_{i j}=\frac{\left(\chi\left(D^{\prime}\right)+\varepsilon\right)}{m n} \sum_{p}^{m} \sum_{p}^{n} \frac{1}{\chi\left(D^{\prime}\right)+\varepsilon}
$$

where $\chi\left(D^{\prime}\right)=\sigma_{G, 1}\left(D^{\prime}\right) \sigma_{G, \xi_{1}}\left(D^{\prime}\right), \xi_{1}$ denotes dimension of filter and $D$ signify guidance picture.

To reach a precise transmission map as well as fine points, the advanced diffusion map split up into smooth and detailed part as

$$
t=t_{\text {smooth }}+t_{\text {det } a i l}
$$

where $t_{\text {smooth }}$ signify smooth part of transmission map while $t_{\text {det ail }}$ denotes detailed part. For smooth part, we obtain Gaussian lowpass filter as effective smoothing filter, and denoted as:

$$
G(d, b)=\frac{1}{2 \pi \sigma^{2}} \cdot e^{\frac{\left(d^{2}+b^{2}\right)}{2 \sigma^{2}}}
$$

where $\sigma$ denotes scale factor of Gaussian blur. Therefore, the smooth and detailed part can estimate as

$$
\begin{aligned}
& t_{\text {smooth }}=t * G \\
& t_{\text {det } a i l}=t-t_{\text {smooth }}
\end{aligned}
$$

Following, the improved transmission map evaluated as:

$$
t_{\text {enhanced }}=t_{\text {smooth }}+\alpha \cdot t_{\text {det } a i l}
$$

where $\alpha$ represents the enhancement factor to manage the improved measure of detailed part.

\subsection{Color Adjustment}

The reflectance part returns the texture and fine points of submerged picture, and only undergoes color deformation caused with energy absorption underneath. Simplest color adjustment [27] is an outstanding means for confirming vast stability among adjustment concert in addition to data loss. The energy absorption in undersea causes color distortion. To overcome this problem, simplest color balance scheme for only extend the picture element of color means whereas conserve data as much as probable with physically set dissimilar truncation relation of color channel, so their histograms are capable towards maximum range [0,255]. For quick stretch, apply an affine transform function on each color channel for mapping picture element from 0 to 255 . Though, only some unusual pels previously map most and least standards, for improving color act with small picture element by peak and least rates prior to affine transform task. In fact, this procedure source extra or less white and black area may seem to be not natural. In general, this algorithm not focuses for proper color allotment, but give color adjustment with contrast to some degree.

\subsection{Hybrid Technique}

A hybrid technique mainly combines dual imagery to generate clear representation. The vital task is mainly for computing weight maps of descriptions furthermore joins them mutually by luminance, saliency along with exposedness weight map for deriving normalized weighted maps [28].

Luminance weight map [29] represents luminance factor of every picture part, created with computing STD among color channels along with luminant rate, where luminant rate $l$ represents:

$$
l=\alpha \cdot r+\beta \cdot g+\gamma \cdot b
$$

where $\alpha+\beta+\gamma=1$, each denotes weight constraint of color part. It is capable for exactly reflect luminant measure of picture and illustrate better improvement; it confirms negative possessions on contrast and colourfulness. Headed for avoiding these, introduces successive weight maps.

Saliency weight-map [30], reflects salient things and points, intends to highlight this discerning submerged sight. Main drawback is for overestimating important area, as a result exposedness weight-map exploits to assure precision as well as defend middle color in picture.

Exposedness weight-map [30], assess significance of exposed picture elements. It protects local dissimilarity be nonexaggerated or non-understated. In general, picture element rates close to average rate are likely to have superior look. Gaussianmodelled remoteness to average rate represents

$$
W_{E}=e^{-\frac{(I(d)-\bar{I})}{2 \sigma^{2}}}
$$

where $\sigma$ denotes standard deviation, $I(d)$ signify pel rate set at location $d$ and $I$ represent mean rate. As a result, the entire weight maps proficient for generating fine look for output imagery. The normalized weight map is simply computes:

$$
W_{\text {norm }}=\sum_{k=1}^{K} W_{k}
$$

where $W_{k}$ specify the kth weight map.

The last free backscatter and color deformation picture be derived as:

$$
R_{c}=\sum_{n=1}^{N} W_{n o r m}^{n} \cdot J_{c}^{n}
$$

where $R_{c}$ denotes color part of fused image, $J_{c}^{n}$ signifies color part of $\mathrm{n}^{\text {th }}$ input. To get enhanced output, we basically exploit a multi-scale pyramid decomposition system for achieving fusion technique. The joint development represents

$$
R_{c}^{l}=\sum_{n=1}^{N} G^{l}\left\{W_{n o r m}^{n}\right\} \cdot L^{l}\left\{J_{c}^{n}\right\}
$$




\section{Jiji et al. / Advances in Science, Technology and Engineering Systems Journal Vol. 4, No. 2, 340-350 (2019)}

where $L^{l}\left\{J_{c}^{n}\right\}$ denotes Laplacian pyramid of color part of $n^{\text {th }}$ input representation, $G^{l}\left\{W_{n o r m}^{n}\right\}$ is $n^{\text {th }}$ stabilized weight map and $l$ represents pyramid stages. While this scheme achieves quite rapid with the enhanced output image can make excellent result.

\section{Experimental Results and Analysis}

The projected technique evaluates the state-of-art processes; by imagery composed as of representational undersea test imagery and dissimilar metrics utilized to compare enhancement performances of these processes.

\subsection{Qualitative Evaluations}

To verify the effectiveness adaptive regularizations, we primarily use reconstruction outcome taking place in picture seer with projected technique in Figure 2. Adaptive regularization shows improved results to remove ringing artifacts near boundaries. The projected technique in Figure 3 composed of adaptive regularization to reconstruct the imagery, weighted decomposition process to separate illuminant and reflecting part. The illuminant part is to improve contrast and texture details using dehazing via gradient guided filter. The reflecting part is to enhance color using color adjustment. Finally, Hybrid technique used to enhance the result. As of Figure 4 weights of dual mechanisms applies a proficient technique for attaining excellence picture. In order to represent different features, we used numerous weight map process to compute the descriptions of true imagery and then join to obtain normalized weight maps. Evaluation of a projected scheme with existing technique shown in Figure 5. The scheme in [21] better reconstructs the picture but fails to enhance its visibility. The scheme in [14] fails to correct and enhance visibility of degraded picture, amplify noise as well as show poor effect on color adjustment. The process in [15] overenhance distorted picture, which causes severe, color distortion along with noise amplification, thus integral performance of enhanced picture is worse. The scheme [30] gets good result, not only correcting the color casting but also enhancing the contrast for removing haze. Though, dissimilarity is overstretched in [24], a few picture elements reduced using over-flow or under-flow. The over-enhancement problem in [25] for test images and some information loss exist as well as in boundary effect due to guided filter introduced. Our projected technique is close towards or even somewhat improved than state of the arts. The RGB color space of dissimilar patch dimension in Figure 6 attains fewer pel map result. Moreover, RGB color space in Figure 7 of original imagery with improved results that all the pels of original picture maps into RGB color space gather in left corner, where is large green rate and small blue and red rate, and other method make more or less stretch of the pixel mapping results. The mapping outcome of our scheme is most excellent and it maps the largest area in the RGB color space, which means it has the best integral dynamic compression range. However, many mapping pixels of our method mapped the boundary, i.e., dark region and brightest region, it indicates that there is information loss of our method.

\subsection{Quantitative Evaluations}

The submerged quality metrics for evaluating and analyzing excellence of underneath imagery, including gray mean, standard deviation, mean gradient etc. The gray mean rate of an image reflects integral intensity, and higher the gray mean is, higher the intensity is, while standard deviation of a picture reflects high frequency part of a picture, which relates picture contrast, higher the standard deviation is, higher the contrast is as well as greater color data. Meanwhile, [31] show that a picture shows good integral quality when gray mean among 100 and 200 and its standard deviation among 35 and 80 after they analyzed and statistic a large measure of imagery. Mean gradient reflects speed of the changes of minor details in picture; it can represent the features of texture transform and the degree of clearness well. Table 1 review metric outcome for reconstruction used for different patch dimension also patch by small dimension offer better result. Following, Table 2 achieves best results in [15], $\mu_{\mathrm{RG}}$ and $\mu_{\mathbf{Y}}$ which are close to zero. The largest $\boldsymbol{\sigma}_{\mathbf{R G}}$ in [25], largest $\sigma_{\text {Yв }}$ in original imagery. Overall, our method obtains the highest score after computing the PSNR, SSIM, UICM, UIConM and UIQM value, lowest score in SSIM. Consequently, projected system is better than existing system giving enhanced outcome.

$$
\begin{gathered}
\text { Mean }=\frac{1}{U V} \sum_{u=1}^{U} \sum_{v=1}^{V} E(u, v) \\
\sigma^{2}=\frac{1}{U V} \sum_{u=1}^{U} \sum_{v=1}^{V}\{E(u, v)-\text { Mean }\}^{2} \\
A G=\frac{1}{U V} \sum_{u=1}^{U} \sum_{v=1}^{V} \sqrt{\frac{[E(u, v)-E(u+1, v)]^{2}+[E(u, v)-E(u, v+1)]^{2}}{2}}
\end{gathered}
$$

Where $\sigma^{2}$ denotes standard deviation, Mean represents mean picture element, $A G$ represents mean gradient and $U V$ signify amount of picture elements. Efficient metric UIQM composed of three independent measurements [32-34] as described below.

a) Undersea Image Colorfulness Measurement (UICM)

The performance of color correction used for evaluating performance of undersea picture improvement progression, with red-green (rg) along with yellow-blue (yb) color parts are used:

$$
\begin{aligned}
& r g=r-g \\
& y b=\frac{r+g}{2}-b
\end{aligned}
$$

Considering that submerged picture often suffers heavy noise, the asymmetric alpha-trimmed statistical standards [35] used for measuring submerged picture colorfulness,

$$
\mu_{r g}=\frac{1}{N-T_{L}-T_{r}} \sum_{x=T_{L}}^{N-T_{r}} \text { Intensity }_{r g}(x)
$$

where $N$ signify total number of pels in the rg part and all pels sorted as $x_{1}<x_{2}<\ldots . .<x_{N}, T_{L}=\alpha_{L} N$ and $T_{r}=\alpha_{r} N$ are the amount of least and most picture element to be reduced as of 


\section{Jiji et al. / Advances in Science, Technology and Engineering Systems Journal Vol. 4, No. 2, 340-350 (2019)}

sorted order $x_{1}<x_{2}<\ldots .<x_{N}$. The average rate $\mu_{r g}$ corresponds to chrominance gray, and average rate close towards nil in rg-yb color part imply superior for balancing color. Further, the secondorder statistic variance is defined by:

$$
\sigma_{r g}^{2}=\frac{1}{N} \sum_{x=1}^{N}\left(\text { Intensity }_{r g}(x)-\mu_{r g}\right)^{2}
$$

$\sigma_{r g}^{2}$ represents the pixel activity. The first and second order statistic information $\mu_{y b}$ and $\sigma_{y b}^{2}$ of the yellow-blue part be computing in similar way. The whole, colorfulness coefficient parameter is expressed as

$$
U I C M=-0.0268 \cdot \sqrt[2]{\mu_{r g}^{2}+\mu_{y b}^{2}}+0.1586 \cdot \sqrt[2]{\sigma_{r g}^{2}+\sigma_{y b}^{2}}
$$

b) Undersea Image Sharpness Measurement (UISM)

Sharpness reflects details and edges of an image, and fine captured images are likely to show better sharpness. For more efficient, enhancement measure estimation (EME) as

$$
E M E=\frac{2}{s . t} \sum_{K=1}^{s} \sum_{L=1}^{t} \log \left(\frac{I_{\max , K, L}}{I_{\min , K, L}}\right)
$$

where picture divided by $s . t$ blocks, and obtain the maximal and minimal pel values in each block, $\frac{I_{\max , K . L}}{I_{\min , K, L}}$ indicates comparative dissimilarity relation in every block. Then the undersea image sharpness measure (UISM) can be written as:

$$
U I S M=\sum_{c=1}^{3} \lambda_{c} \cdot E M E\left(\text { grayscale edge }_{c}\right)
$$

where $\lambda_{c}$ stands for weight coefficient of each color part.

c) Undersea Image Contrast Measurement (UICM)

Intended for submerged descriptions, dissimilarity Distortion is usually happened with backscattering. The contrast performance be measured by:

$\log A M E E=\frac{1}{s . t} \sum_{K=1}^{s} \sum_{L=1}^{t} \frac{I_{\max , K, L}-I_{\min , K, L}}{I_{\max , K, L}+I_{\min , K, L}} \cdot \log \left(\frac{I_{\max , K, L}-I_{\min , K, L}}{I_{\max , K, L}+I_{\min , K, L}}\right)$

and submerged picture contrast measure be written as:

$$
\text { UIConM }=\log \text { AMEE(Intensity) }
$$

d) Undersea Image Quality Measurement (UIQM)

Undersea descriptions modelled as linear superposition of absorbed as well as spreaded part. Meanwhile, absorption and scattering by dusk-like particles causes color casting, sharpness attenuation with dissimilarity distortion. Consequently, logical use of linear form to create entire UIQM as:

$$
U I Q M=\alpha \cdot U I C M+\beta \cdot U I S M+\gamma \cdot U I C o n M
$$

where $\alpha, \beta$, and $\gamma$ signifies weight coefficients to organize each measure as well as balance their values.

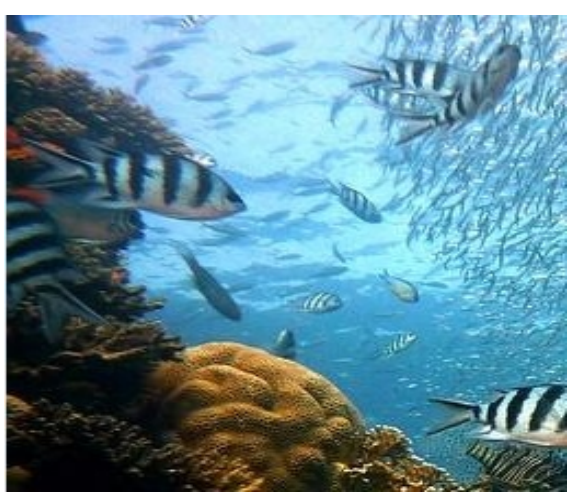

a) True Picture

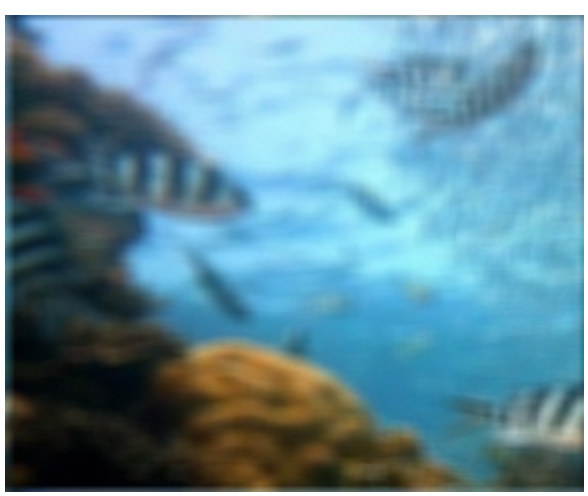

b) Blurred

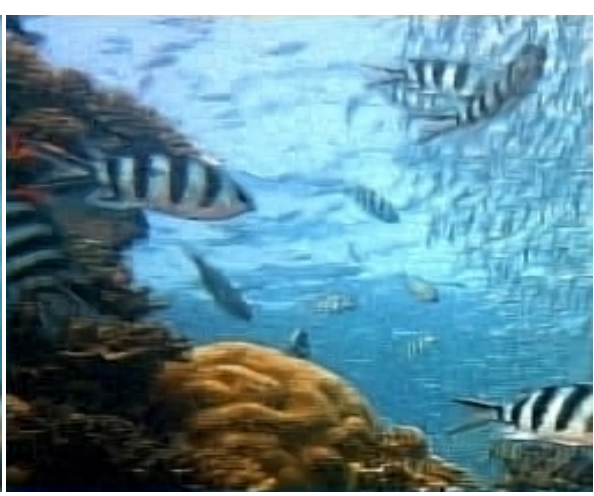

c) Restored Picture

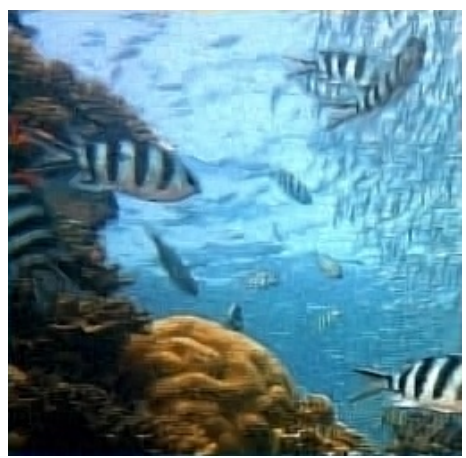

a) Reconstructed Image

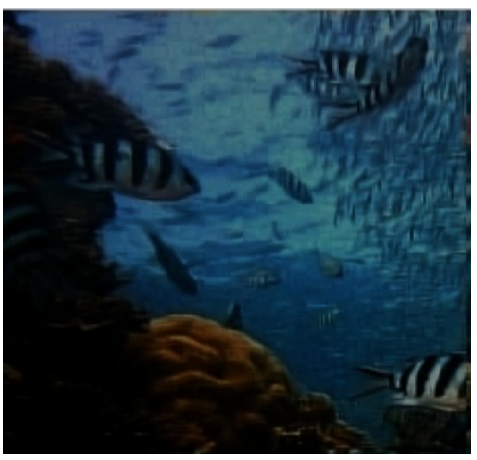

b) Reflectance

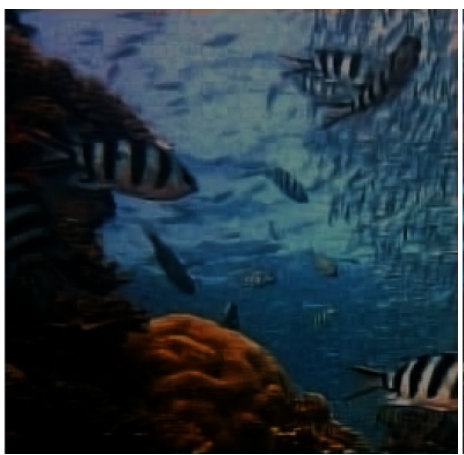

c) Color Balance

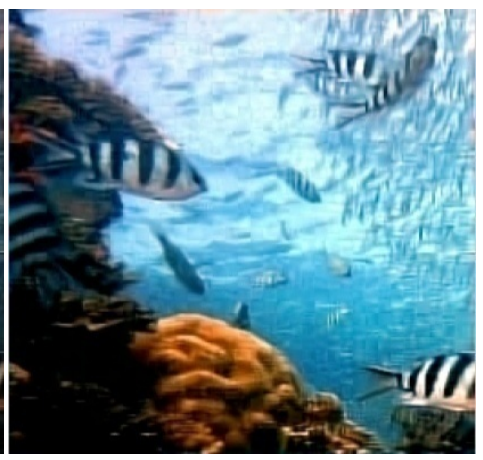

f) Final Output 
C. Jiji et al. / Advances in Science, Technology and Engineering Systems Journal Vol. 4, No. 2, 340-350 (2019)

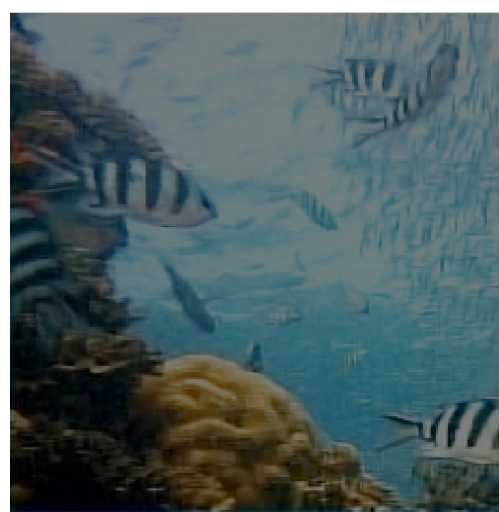

d) Illuminance

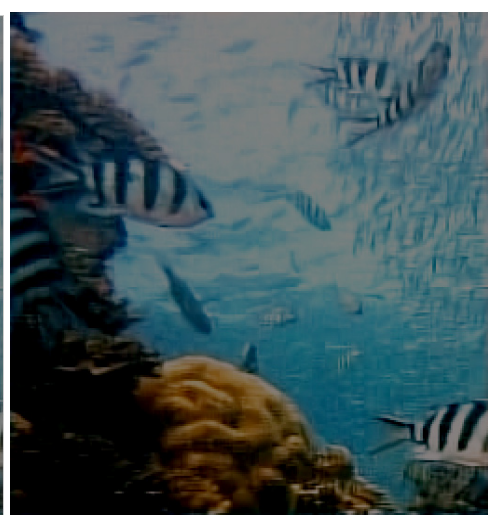

e) Dehazing viagradient guided filter

Figure 3: Results on the process of Proposed Method

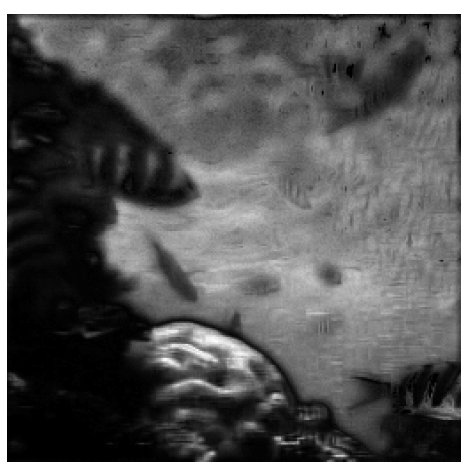

a) Luminance weight 1

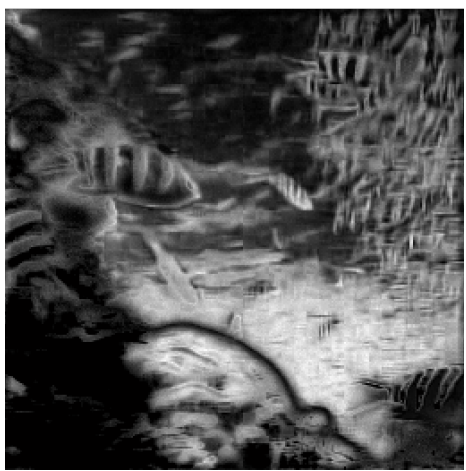

e) Luminance weight 2

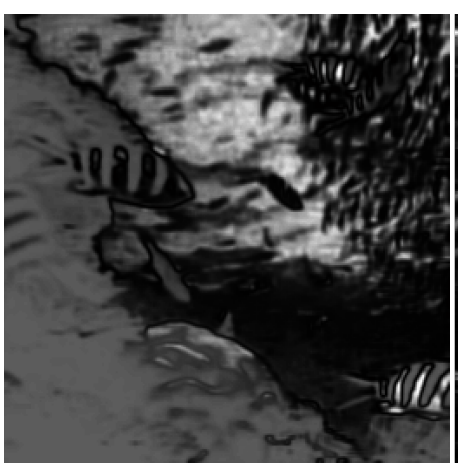

b) Saliency weight 1

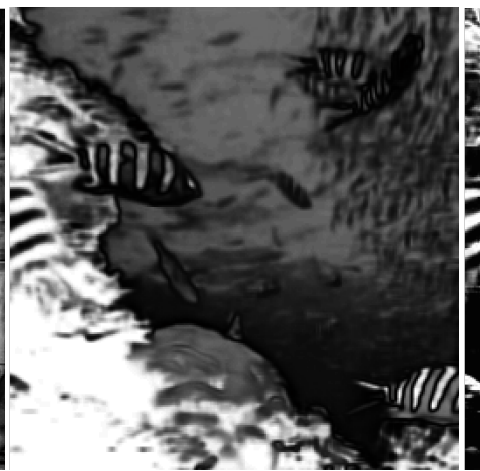

f) Saliency weight 2

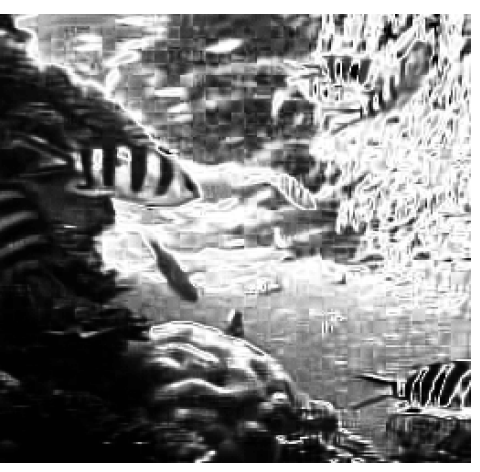

c) Exposedness weight 1

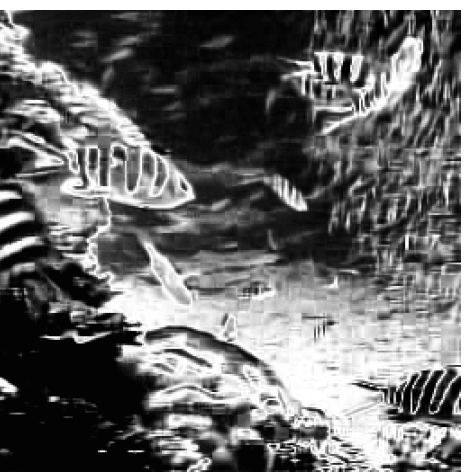

g) Exposedness weight 2

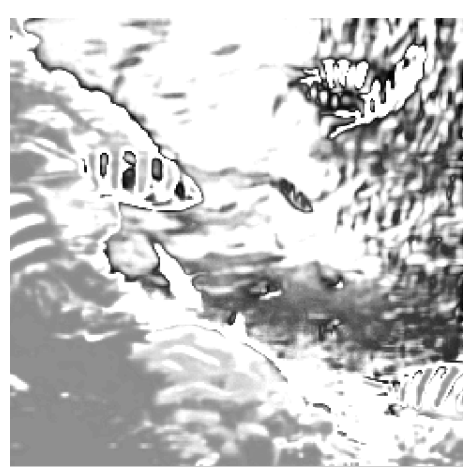

d) Normalized weight 1

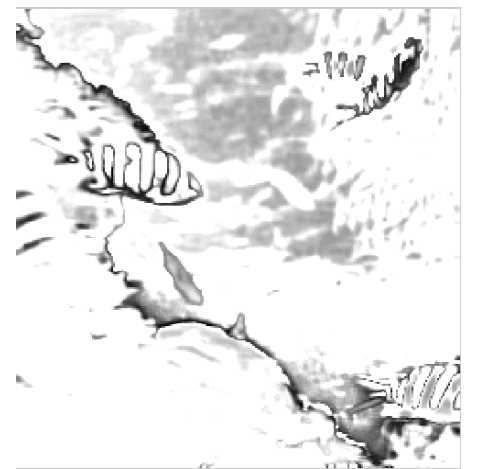

h) Normalized weight 2

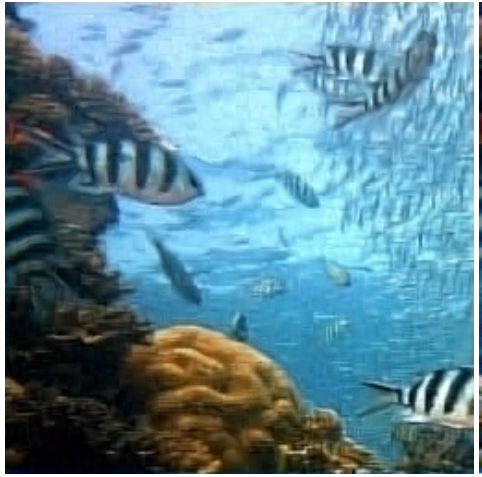

a) [21]

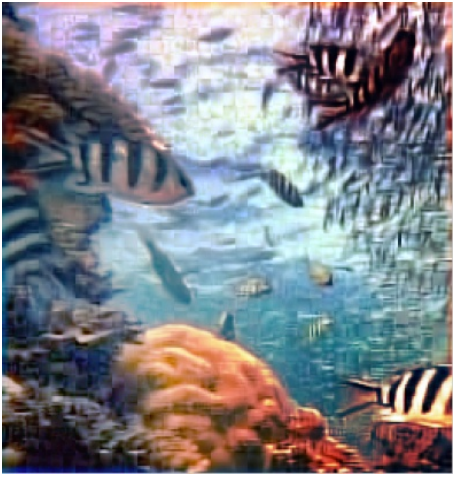

b) [14]

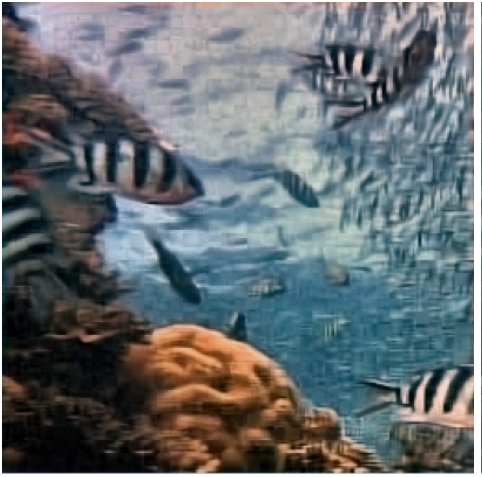

c) [30]

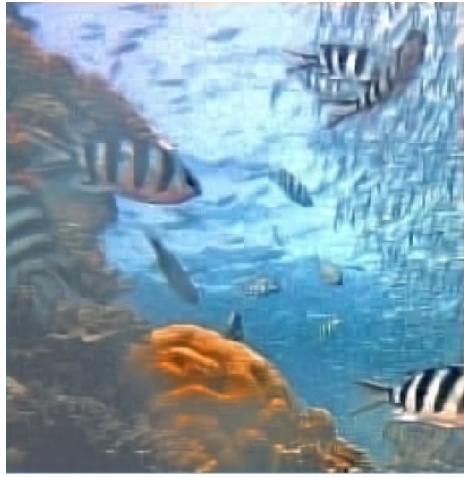

d) [24] 
C. Jiji et al. / Advances in Science, Technology and Engineering Systems Journal Vol. 4, No. 2, 340-350 (2019)

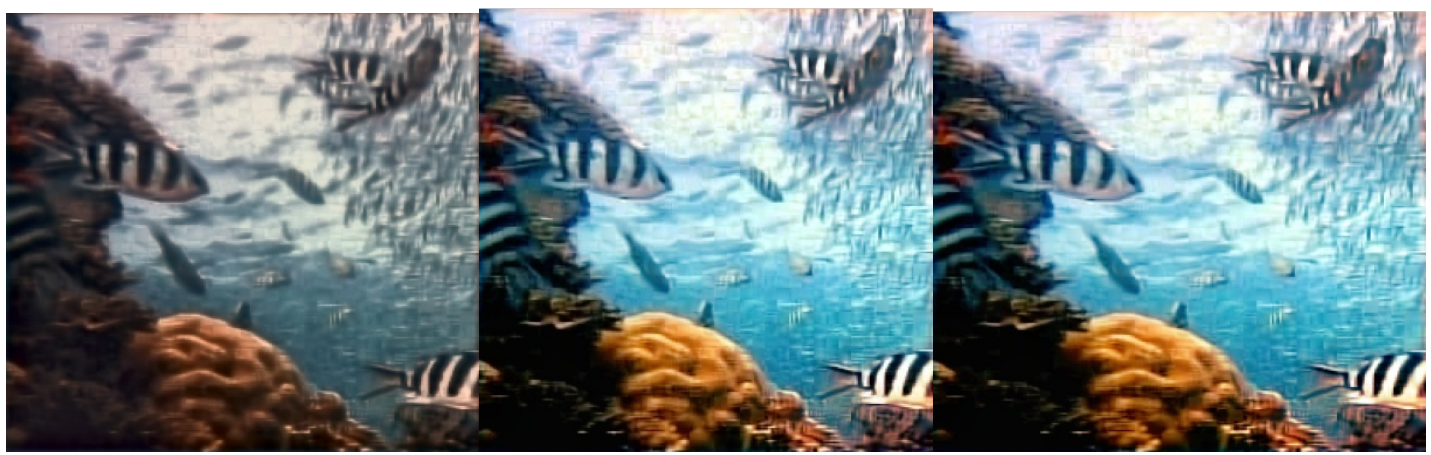

e) [15]

f) $[25]$

g) Ours
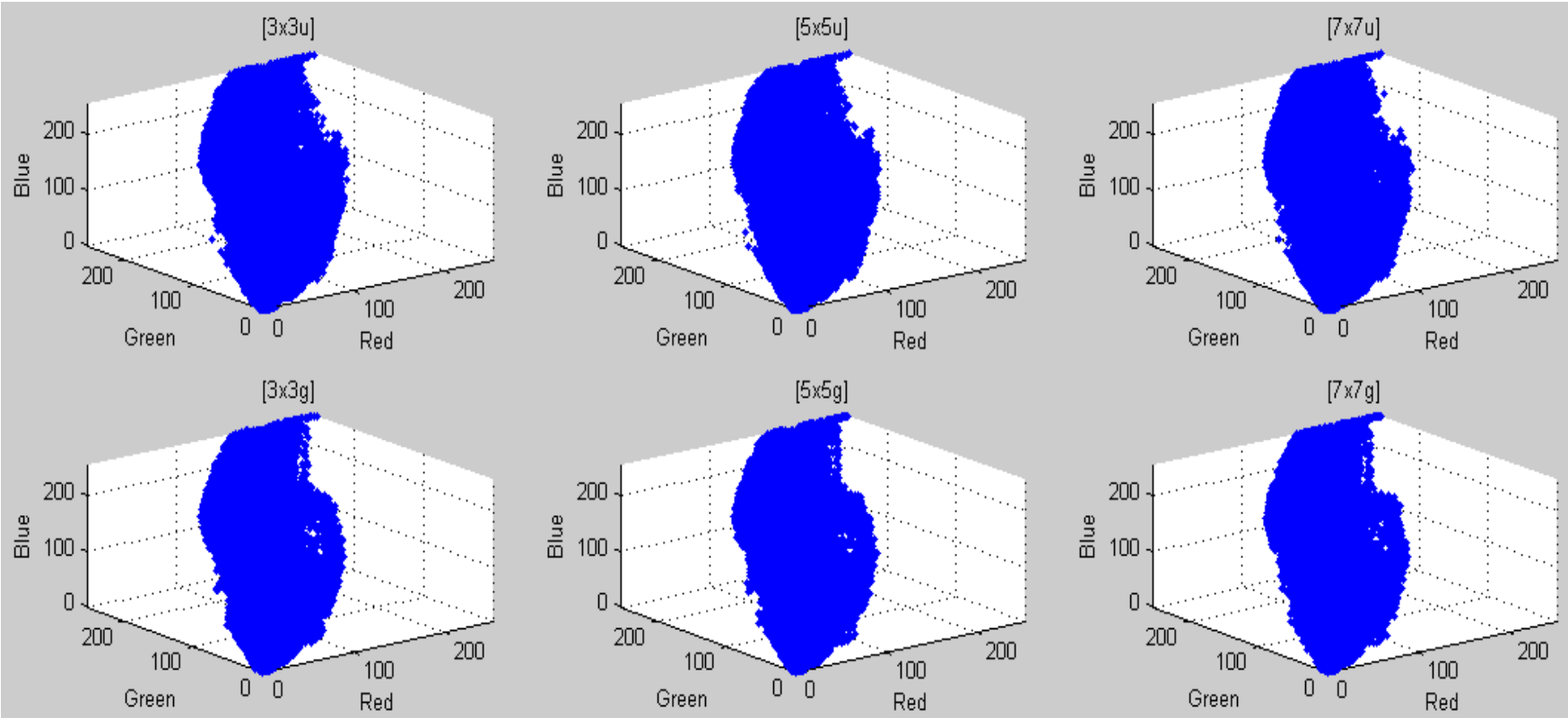

Figure 6: RGB color space mapping reconstruction results on seer image
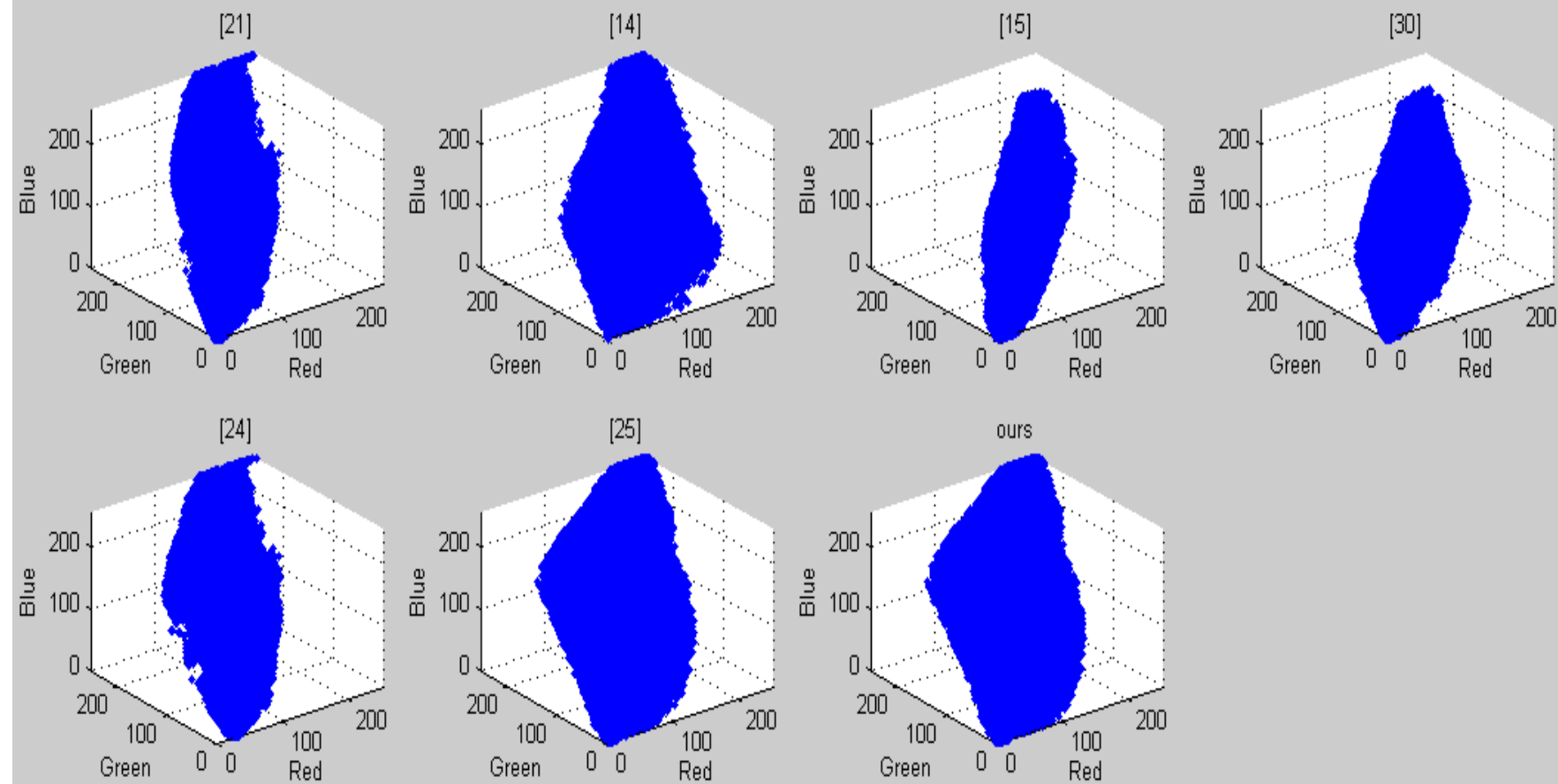

Figure 7: RGB color space mapping Enhancement results on seer image 
Table 1: Reconstruction metric results of seer image

\begin{tabular}{|l|c|c|c|c|c|c|}
\hline \multirow{2}{*}{} & \multicolumn{3}{|c|}{ Uniform blur } & \multicolumn{3}{c|}{ Gaussian blur } \\
\cline { 2 - 7 } Mean & $\mathbf{7 \times 7}$ & $\mathbf{5 \times 5}$ & $\mathbf{3 \times 3}$ & $\mathbf{7 \times 7}$ & $\mathbf{5 x 5}$ & $\mathbf{3 \times 3}$ \\
\hline SD & 127.33 & 127.35 & 127.36 & 127.35 & 127.37 & 127.37 \\
\hline AG & 71.266 & 71.184 & 71.136 & 70.148 & 70.065 & 70.027 \\
\hline PSNR & 26.16 & 26.28 & 26.35 & 23.96 & 24.04 & 24.05 \\
\hline RMSE & 17.827 & 17.469 & 17.270 & 13.689 & 13.320 & 13.169 \\
\hline SSIM & 0.741 & 0.754 & 0.756 & 0.618 & 0.623 & 0.622 \\
\hline $\boldsymbol{\mu}_{\text {RG }}$ & -45.531 & -45.543 & -45.558 & -45.535 & -45.538 & -45.537 \\
\hline $\boldsymbol{\mu}_{\text {YB }}$ & 27.93 & 27.92 & 27.906 & 27.734 & 27.73 & 27.729 \\
\hline $\boldsymbol{\sigma}_{\text {RG }}$ & -41.214 & -41.311 & -41.352 & -41.272 & -41.326 & -41.34 \\
\hline $\boldsymbol{\sigma}_{\text {YB }}$ & 27.004 & 27.038 & 27.052 & 26.892 & 26.91 & 26.918 \\
\hline UICM & -45.531 & -45.543 & -45.558 & -45.535 & -45.538 & -45.537 \\
\hline UIConM & 0.745 & 0.737 & 0.736 & 0.716 & 0.704 & 0.704 \\
\hline UISM & 7.059 & 6.890 & 6.906 & 6.512 & 6.276 & 6.244 \\
\hline UIQM & 3.467 & 3.388 & 3.389 & 3.199 & 3.0891 & 3.080 \\
\hline
\end{tabular}

Table 2: Enhancement metric results of seer image

\begin{tabular}{|l|c|c|c|c|c|c|c|c|}
\hline & Original & {$[\mathbf{2 1}]$} & {$[\mathbf{1 4}]$} & {$[\mathbf{3 0}]$} & {$[\mathbf{2 4}]$} & {$[\mathbf{1 5}]$} & {$[\mathbf{2 5}]$} & Ours \\
\hline Mean & 127.39 & 127.34 & 129.26 & 104.05 & 128.99 & 103.41 & 136.21 & $\mathbf{1 3 6 . 2 1}$ \\
\hline SD & 66.272 & 71.236 & 68.047 & 56.586 & 70.056 & 64.509 & 82.112 & $\mathbf{8 3 . 4 9 4}$ \\
\hline AG & 3.790 & 9.226 & $\mathbf{1 5 . 8 1 9}$ & 11.89 & 9.326 & 8.660 & 12.212 & 12.517 \\
\hline PSNR & 21.53 & 24.10 & 27.670 & 28.483 & 16.557 & 28.073 & 30.004 & $\mathbf{3 0 . 0 1 3}$ \\
\hline RMSE & 21.369 & 13.717 & 10.544 & 9.602 & 37.900 & 10.022 & 8.059 & $\mathbf{8 . 0 5 2}$ \\
\hline SSIM & 0.466 & 0.619 & 0.724 & 0.786 & 0.839 & 0.765 & 0.841 & $\mathbf{0 . 8 4 2}$ \\
\hline $\boldsymbol{\mu}_{\text {RG }}$ & -45.685 & -45.533 & -4.937 & -24.524 & -33.344 & $\mathbf{- 0 . 9 6 0}$ & -37.795 & -37.801 \\
\hline $\boldsymbol{\mu}_{\text {YB }}$ & -41.664 & -41.224 & -13.078 & -25.712 & -36.852 & $\mathbf{- 1 . 7 3 2}$ & -35.456 & -35.472 \\
\hline $\boldsymbol{\sigma}_{\text {RG }}$ & 27.789 & 27.93 & 22.343 & 23.581 & 33.732 & 11.964 & $\mathbf{2 9 . 8 9 6}$ & 29.888 \\
\hline $\boldsymbol{\sigma}_{\text {YB }}$ & $\mathbf{2 7 . 1 5 5}$ & 27.016 & 20.332 & 22.625 & 30.378 & 9.647 & 26.214 & 26.226 \\
\hline UICM & 4.505 & 4.516 & -4.937 & 4.223 & -33.344 & -0.960 & 4.917 & $\mathbf{4 . 9 1 7}$ \\
\hline UIConM & 0.621 & 0.741 & 0.836 & 0.827 & 0.790 & 0.872 & 0.480 & $\mathbf{1 . 0 3 1 8}$ \\
\hline UISM & 5.771 & 7.005 & $\mathbf{7 . 1 3 2}$ & 7.055 & 7.003 & 6.911 & 7.013 & 6.997 \\
\hline UIQM & 2.637 & 3.434 & 4.957 & 4.3512 & 3.952 & 5.132 & 2.723 & $\mathbf{4 . 6 8 9}$ \\
\hline
\end{tabular}

\section{Conclusion and Future Work}

This work is mainly for Enhancing undersea representation in blurry conditions using Hybrid technique. Our scheme composed with Adaptive regularization for recreation, weighted decomposition procedure to separate recreated imagery into color change along with dehazed schemes separately, then multi-scale fusion technique towards enhancing picture. The strong with effective Hybrid technique which incorporate the description of all fused imagery into resultant outcome constitute backbone of projected scheme. In fact, the projected scheme generally beats other methods in terms of qualitative as well as quantitative evaluations. Our technique confirms excellent act for undersea imagery, but border effect cause data loss. Meantime, our technique does not outperform some of the state-of-art system and even direct over improvement for precise cases. The challenging www.astesj.com work for future would be to find out a well-organized means to about vertical deepness and optimize the transmission deepness of undersea imagery more precise, at last build up absolute, effective and well-organized system.

\section{Conflict of Interest}

The authors declare no conflict of interest.

\section{Acknowledgment}

The authors thank the reviewers for their thorough and helpful remarks.

\section{References}

[1]. Ruo W, Yaf W, Jun Z, Xianp F. "Review on Underwater Image Restoration and Enhancement Algorithms", 7th Intern Confrnc on Intrnt Multimedia Comput and Serv ACM, 2015. 


\section{Jiji et al. / Advances in Science, Technology and Engineering Systems Journal Vol. 4, No. 2, 340-350 (2019)}

[2]. Schetni R, Cors S. "Underwater Image Processing: State of the Art of Restoration and Image Enhancement Methods", EURASIP Jrn Advn sgnl Pr, Jan 2010.

[3]. John C, Yin C.C. Underwater Image Enhancement by Wavelength Compensation and Dehazing, IEEE Transc Img Pr-2012.

[4]. C. Ancut, Hermns, and Beka, "A Fast Semi Inverse Approach to Detect and Remove the Haze from a Single Image',' Comp Vis: Springer, Nov2011

[5]. O. Ancu, C. Anc, and Bekae," Effective Single Image Dehazing by Fusion," Procd IEEE Intr. Confr. Img Proc, Sep-2010.

[6]. Ancut and Ancut C, "Single Image Dehazing by Multi-Scale Fusion," IEEE Transct Img Proc, Aug-2013.

[7]. C. O. Ancu, T. Habe, and Bekae, "Enhancing Underwater Images and Videos by Fusion," Proce. IEEE Confrn. Comp. Visn. Pattrn Recogn, Jun. 2012.

[8]. K. Hem, Jia S, and Xiao T, "Single Image Haze Removal using Dark Channel Prior,", IEEE Transc. Patrn Analy. Macnn, Dec. 2011.

[9]. Raan F, "Single Image Dehazing", ACM -Transc Graphic, Aug- 2008.

[10]. Xue F, Yu H, Del Z, Xia P and Xing D, "A Fusion-based Enhancing Approach for Single Sandstorm Image", IEEE Intrn. Wrkshp Mult S/gl Proc, Sept-2014.

[11]. Fan G, Jin T and Zix C, "Fusion Strategy for Single Image Dehazing", Intrn. Jrn. Dig. Contt Techl. App, Jan. 2013.

[12]. C. O. Ancuti, C. Ancuti, C. De Vleeschouwer, and A. C. Bovik, "Singlescale fusion: An effective approach to merging images," IEEE Trans. Image Process., vol. 26, no. 1, pp. 65-78, Jan. 2017.

[13]. H. Lu, Y. Li, S. Nakashima, H. Kim, and S. Serikawa, "Underwater image super-resolution by descattering and fusion," IEEE Access, vol. 5,pp. 670679, 2017.

[14]. Zuiderv K., "Contrast limited adaptive histogram equalization", pub book Graphcs gem IV, 1994.

[15]. Fu X, Zhug P, Huang Y, Yin L, Xia P and Xing D, "A Retinex-based Enhancing Approach for Single Underwater Image", Img Procd IEEE Intrl Confr, 2014

[16]. Emb S., Chi L., Cava A, "Underwater image and video dehazing with pure haze region segmentation", Comp Vis and Im Unde, 2018.

[17]. Peng Y, Cos P,"Underwater image restoration based on image blurriness and light absorption",IEEE Tran on Im Pro, 2017.

[18]. Li C, Cava, "Background light estimation for depth-dependent underwater image restoration",IEEE Intl Conf on Ima Processg, 2018.

[19]. AncuC., Ancut., D Vlee, Bekt, P, "Color balance and fusion for underwater image enhancement" IEEE Transa Imge Pro, 2018.

[20]. Ber D., Trei T., Avid S, "Diving into haze-lines: Color restoration of underwater images", Brith Mach Visn Confer, 2017

[21]. Chrispin jiji and Nagaraj Ramro "Deblurring Underwater Image Degradations based on Adaptive Regularization" IEEE Inter Conf on Computat Intelli and Comput Rese, 2017

[22]. Weis D, Guang S, Xi L, Lei Z and Xiaol, "Image Reconstruction with Locally Adaptive Sparsity and Nonlocal Robust Regularization", S/gl Procg: Imag Comn, Nov-2012.

[23]. Dong, Zhan, Gua S and Xiaoli, "Image Deblurring and Super Resolution by Adaptive Sparse Domain Selection and Adaptive Regularization", IEEE Transa Ime Proces, July 2011.

[24]. Kwa K, Wo J, You S "Optimized Contrast Enhancement for Real Time Image and Video Dehazing”, Jrn Visu Commn Img Repr, Feb-2013.

[25]. Zhang, "Removing backscatter to enhance the visibility of underwater objects",msc dissertn, Nanyang Univ, 2016

[26]. F Ko, Weih C, Changy W, Zhengg L, "Gradient Domain Guided Image Filtering", IEEE Transc Img Proceg, 2015.

[27]. Cod. Ancut, Cosm Chri, Philip, "Color Balance and Fusion for Underwater Image Enhancement", IEEE Tra Img Procg, Jan 2018

[28]. C.Anc, Ancu Cod, T Hab, Phil B,"Fusion-based Underwater Image Enhancement by Weight Map Techniques",IC3-2018,Chap in Adv in Intell Sys and Comp, Jan 2019.

[29]. Ancti, Habr T and Phil B. Fusion-based Restoration of the Underwater Images", Intrn Confrn Img Proc, Sep-2011.

[30]. Ancti C, Ancu O, Haer "Enhancing Underwater Images and Videos by Fusion", Compr Visn Patn Recogn, Jun-2012.

[31]. Jobson D, Rahman Z, Woodel "The Sstatistics of Visual Representation", Procd SPE- 2002.

[32]. Panet $\mathrm{K}, \mathrm{Ga} \mathrm{C}$ and Agai S,"Human-Visual-System-Inspired Underwater Image Quality Measures”, Jr of Ocen E-2016.

[33]. Cha Y, Ric M, Andr C, “An Online Platform for Underwater Image Quality Evaluation", Intl Confr on Patn, CPR 2018

[34]. Priya S and Sim, "Quality measure technique for Underwater images using Artificial neural network" Jon of Eng and Appl Sc, 2018
[35]. Bedr and Watt, "Alpha-Trimmed means and their relationship to Median Filters," IEEE Transc Acost. Spch Sigl Proc, 1984. 